Indonesian Journal of Electrical Engineering and Computer Science

Vol. 12, No. 1, October 2018, pp. 218 224

ISSN: 2502-4752, DOI: 10.11591/ijeecs.v12.i1.pp218-224

\title{
VANETs and Internet of Things (IoT): A Discussion
}

\author{
Shahirah Mohamed Hatim, Shamsul Jamel Elias, Norkhushaini Awang, Mohd Yusof Darus \\ Faculty of Computer and Mathematical Sciences, Universiti Teknologi MARA, Shah Alam, Malay sia
}

\begin{tabular}{|c|c|}
\hline Article Info & ABSTRACT \\
\hline Article history: & \multirow{6}{*}{$\begin{array}{l}\text { Recent advancement of wireless technology and Internet of Things (IoT) } \\
\text { have brought a significant development in Vehicular Ad hoc Networks } \\
\text { (VANETs). VANET and IoT are the key elements in the current Intelligent } \\
\text { Transport System (ITS). Various research on VANET and IoT shows that } \\
\text { both have substantial effects in smart transportation system. This paper aims } \\
\text { to discuss and illustrate the main challenges and drawbacks; the routing } \\
\text { protocols, security and privacy experienced by VANETs respectively. This } \\
\text { paper also would like to address the importance of IoT based on VANET in } \\
\text { traffic control management system to cope up with the new wireless } \\
\text { technology era. }\end{array}$} \\
\hline Received May 1, 2018 & \\
\hline Revised Jul 10, 2018 & \\
\hline Accepted Jul 25, 2018 & \\
\hline Keywords: & \\
\hline $\begin{array}{l}\text { VANETs } \\
\text { IoT }\end{array}$ & \\
\hline
\end{tabular}

Copyright $(0) 2018$ Institute of Advanced Engineering and Science. All rights reserved.

Corresponding Author:

Shahirah Mohamed Hatim,

Department of Faculty of Computer and Mathematical Sciences,

Universiti Teknologi MARA,

Shah Alam, Malaysia.

Email: shahirah88@uitm.edu.my

\section{INTRODUCTION}

Nowdays, the number of vehicles on the road has been increased rapidly, thus leads to traffic congestion. Traffic congestion can be defined as the consequence of the imbalance traffic flow and the capacity. This situation occurs when the number of vehicles circulates on a specific zone increases to the peak level and exceeds the limit of possible capacity [1], [21]. Technologies that are based on computerized mechanism which are able to distribute the traffic information to the road drivers are needed to overcome the problem. Vehicular Ad hoc Networks (VANETs) is one of the solution that is widely used in various domain of vehicle industry as the communication pace among vehicles and road side units (RSUs). VANETs is a self-organizing network, which is a sub-class of Mobile Adhoc Networks (MANETs) [1-5], [23-24]. It used vehicles as mobile nodes. These vehicles act as the intelligent machine to collect and process data from devices, connected to the Internet by various transmissions through system such as Global Positioning System (GPS) [1], [3], [4].

VANETs interconnected wirelessly via embedded On Board Units (OBUs) wireless device. OBUs act as a platform which permits vehicle to form a short range of wireless ad-hoc networks with the capability of disseminating data and application to vehicular networks [14], [23], [25]. Apparently, VANETs has been successfully supported route safety-related applications and surveillance services, and expected to assist in traffic alert dissemination, context-aware infotainments and mobile vehicular cloud services [1], [4], [6-7]. 


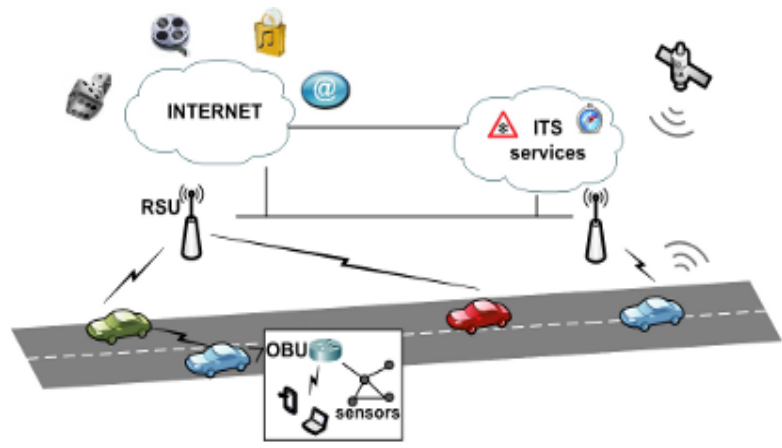

Figure 1. Basic VANETs and IoT [8]

Intelligent Transport System (ITS) is important in supporting traffic safety and traffic efficiency applications [24-25]. It is an important component in developing smart city, where it supports communications between vehicles in the network [1-2], [25], [28], [31]. ITS comes with the capacity to predict, observe and manage traffic movement [5].

Thus, VANETs help ITS in accommodating Vehicle-to-Vehicle (V2V), Vehicle-to-Base Station and Vehicle-to-Infrastructure (V2I) communications which are standardized by IEEE WAVE, standardized for short range communication [1-2], [25], [28]. However, VANET are usually integrated with other algorithms or paradigm such as Dijkstra algorithm, Software Defined Networking, fog computing, Taguchi method etc., due to the challenges differ from the existing VANETs systems, such as inflexibiltity, non-intelligence and broadcast network congestion (excessive amount of broadcast messages) which lead to network data loss [1], [9], [23].

Network with vehicles which can interact with other vehicles and RSUs can be established with the aid of Internet of Things (IoT) [9]. IoT has been increasingly a growing topic in the area of road safety which produces great impacts on society and their life. IoT network connects millions of components, from human to small non-living things. With the support of IoT, waste can be reduced and efficiency can be increased since transportation networks involve the usage of energy [10-11].

\section{VANETS CHALLENGES}

Previous research have shown that VANETs suffers from several challenges; which need to be highlighted, due to the possibility of increasing number of connected devices in the system, resources utilization, unbalanced traffic flow, geographical circumstances, unreliable network connectivity and broadcast storm [1], [19], [26]. However, this paper aims to address the issues of routing protocols and security and privacy in VANETs.

\subsection{Routing Protocols in VANETs}

Routing is the process of transferring packets from a network to the other. VANET uses a wireless based technology for communication between apps and services [3], [5], [23]. It also equipped with impressive characteristics; high dynamic topology and support irregular connectivity [2-3], [20]. However, the initial VANET is not equipped with the routing algorithms which lead to the communication problem among networks [3]. There are various routing protocols that have been applied in the existing VANETs which are categorized into three different types; broadcas t, geocast, and unicast [2-3], [20].

\subsubsection{Broadcast}

This type of protocols is essential in VANETs since there is a need of disseminating message to unknown destination [3]. The recent message broadcast protocols on vehicular ad hoc networks, such as a spatially aware packet routing algorithm, predicts the permanent topology holes and conducts the geographic forwarding, whereas DV-CAST based protocols search the best path to forward the packet [3].

\subsubsection{Geocast}

Geocast routing is based on specified location. It is established with the aim of delivering the packet from source node to all other nodes within a specified geographical region [32]. This type of routing is also considered as multicast routing where it defines a forwarding zone where it directs the packets flooding in the network to reduce message overhead and congestion [32]. Example of geocast protocols available are distributed robust geocast protocol (DRG) and robust vehicular routing (ROVER) [2, 3]. 


\subsubsection{Unicast}

The unicast communication protocols for VANETs can be represented in three ways which are greedy, opportunistic and trajectory-based. Through greedy, nodes forward the packets to their outermost neighbors towards the destination [3], [25]. Example of routing protocols for greedy is greedy traffic-aware routing (GyTAR) [3]. For opportunistic, nodes employ the carry-toward technique in order to speculatively deliver the data to the destination; whereas trajectory based allows nodes calculate possible paths to the destination and deliver the data through nodes along one or more of those paths such as trajectory -based data forwarding (TBD) [3], [27]. Table 1 shows the example of routing protocols in existing VANETs.

Table 1. Routing protocols in existing VANET [2], [3]

\begin{tabular}{ll}
\hline Routing approaches & \multicolumn{1}{c}{ Routing protocols } \\
\hline Unicast & Ad hoc On-Demand Distance Vector (AODV) \\
& General Packet Radio Services (GPRS) \\
& Vehicle-assisted data delivery (VADD) \\
& Dynamic Source Routing (DSR) \\
& A-START \\
& Position-based multi-hop broadcast (PMB) \\
& Improved greedy traffic-aware routing (GyTAR) \\
& Trajectory-based data forwarding (TBD) \\
& Connectivity Aware Routing (CAR) \\
& Geographical Source Routing (GSR) \\
& Opportunistic packet relaying in disconnected vehicular ad \\
& hoc networks (OPERA) \\
& MaxProp \\
& SiFT \\
& Trajectory-based data forwarding (TBD) \\
& Linkage Protocol for Highway automation (DOLPHIN) \\
& BROADcast COMMunication protocol(BROADCOMM) \\
Distributed vehicular broadcast (DV-CAST) \\
Packet routing algorithm \\
Distributed robust geocast protocol(DRG) \\
Robust vehicular routing (ROVER) \\
\hline
\end{tabular}

Routing protocols is very important to ensure scalability but few focused on introducing routing protocols for communication of data dissemination [3]. This challenge can be overcome by integrating IoT in the VANET [1,9].

\subsection{Security and Privacy}

Most of the researchers in the area found that security and privacy are the biggest challenge in transmitting data or messages in VANETs. Kinematic data of the components involved in VANET cannot be exposed to collusion between malicious servers and the users due to the act of online data integrity of personal information [2-3], [5], [12]. Recently, the issues regarding data ownership, massive data management and legal and liability also have been addres sed in the area [12].

In order to overcome the is sue, security architecture plays an important role in ensuring security and privacy. Thus, vehicular communication system structure should focus on providing communication scheme for safety-based application as it will produce share session key for a secure communication in the network [3]. A low security level of data clouds might cause roadside attackers to make fake requests on road or parking cloud services which may lead to confusion [12].

Other issues that have been slightly addressed in existing VANETs research are fundament limits and opportunities in terms of the theoretical perspective [3], [5], [12], the suitable IEEE standards, connectivity between vehicles and the infrastructures, cross-layer design, mobility, validation and cross layer design [3], [12].

\section{VANETS AND IOT APPLICATIONS}

As mentioned in section I, VANET with the aid of IoT has been widely applied for data or message dissemination of safety-based as well as non-safety-based applications to ensure traffic safety and efficiency through broadcasting protocol [23]. It derives the idea of building smart cities where VANET systems are 
interconnected to IoT in terms of keeping the data on the internet, or also known as cloud. The concept of smart city is important to increase the quality of services (QoS) provided to citizens and eventually improve their quality of life in this new era of technology [30]. Table 2 shows the relationship or the needs between VANET and IoT. It can be said that both are interrelated to each other.

Table 2. VANET and IoT applications

\begin{tabular}{ll}
\hline VANET Application & IoT Application \\
\hline Intersection collision warning & Smart cities \\
Lane change assistance & Environment monitoring \\
Road map & Energy management \\
Accident detection and alerting & Medical healthcare system \\
Traffic planning & Building automation \\
Overtaking vehicle warning & Transportation \\
Emergency vehicle warning & Social network \\
Point of interest (PoI) allocation & \\
Weather information & \\
\hline
\end{tabular}

\subsection{Safety Applications}

Accident prevention is the traditional intention of safety applications, thus lead to the development of vehicular adhoc network where communications among the related components in the environment are involved. [2,3]. These applications provide life-saving traffic assistance to drivers on the road [2, 25]. It is categorized into three main categories which are driver assistance, alert information and warning alert [2].

\subsection{Non-safety Applications}

Non-safety applications are also important in ensuring traffic convenience and efficiency when we are on the road. Information on weather, the location and current traffic movements on the road networks, distance, Point of Interest (PoI) allocation as well as social network (connected to mobile network through smartphones) can be beneficial to the road users connected in the VANET systems using IoT [2, 3, 22]. With the aid of non-safety applications, it can help safety applications to work better and increase efficiency to avoid accidents.

\section{RELATED WORKS}

Due to the weakness of the existing VANET, some research have tried to emerged networking paradigms such as Software Defined Networking (SDN); proposed for wired environment with Fog Computing (for real-time processing purpose) since Fog is considered as a cloud server known as IoT to provide attractive approach in existing VANET [1]. SDN can enable VANET to alter the network topology changes [5]. The proposed architecture by combining safety through data streaming and non -safety services through lane-change assistance, managed to reduce the latency of the VANET.

VANET also has been proved to work for Smart Accident Management System [9] where the authors combine IoT and VANET to propose a new framework for automated accident alerting and traffic optimization. Surprisingly, the combination produces great result which allows the VANET entities vehicles, ambulance and hospitals to sustain the network themselves. In this framework, ambulance can receive the information on the shortest path (using $\mathrm{A}^{*}$ algorithm) to the accident zo ne and also obtain the information on the nearest nearby hospital through GPS and the cloud server whereas hospital can prepare for the suitable treatment before the patient reach the hospital according to the patient's condition signal sent by the ambulance [9]. The system organization is illustrated in Figure 2.

IoT in VANET has also successfully applied in real-time decision making for IoT-based traffic light control using game theoretical approach whereby the authors proposed an approach to control congestion or traffic flow at intersections [13]. Their approach is able to identify the priority vehicles that need to be treated immediately such as patrol car, ambulance and fire engines, thus improve the average waiting time of these priority vehicles at intersections [13]. There is a serious need for this type of system due to the urbanization growth day to day.

The idea in [13] is the same as in [14] where both system proposed to solve congestion of traffic flow. A city lane and intersection model has been proposed to manage the challenge of vehicle mobility. As a result, the system is able to improve congestion and road safety [14]. 


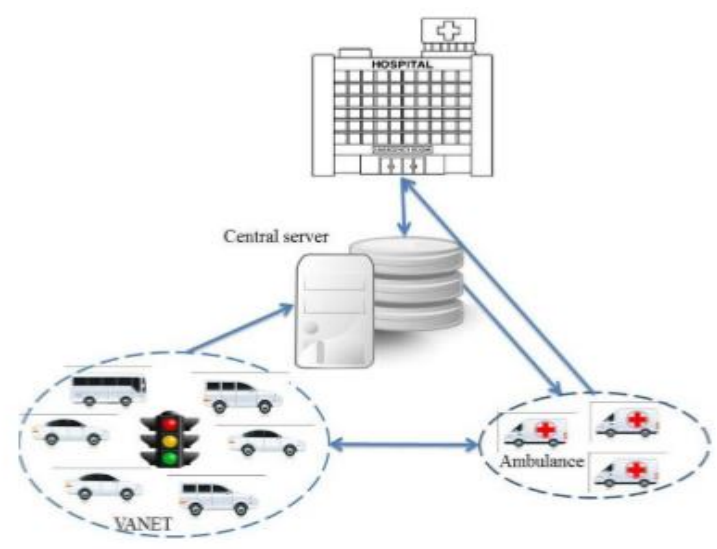

Figure 2. System organization [7]

Similarly, authors in [5] discussed on the importance of Vehicular Cloud Computing in VANET for traffic control management. Traffic congestion creates more negative consequences. It affects their daily activities, their safety and their quality of life, thus attract many different scientific interests [5, 16]. Road traffic safety (RTS) must be the essential is sue in any urban environment planning [5, 17]. The author also addressed the main cause of today's road congestion is that the number of circulating vehicles in a specific zone increases and exceeds its critical capacity [5, 17, 21]. Current developments in the field of Command, Control, Communications, Computers and Intelligence (C4I) technologies have directed to a wide growth in all the intelligent devices by equipping them with wireless communication technology and embedded processors [5]. These smart devices can be utilized to offer a suitable and better environment by forming new useful era of Internet of Things (IoT) [5, 17].

In [25], the authors proposed a scalable congestion control focus on disseminating event-driven safety messages in VANETs. In dense network, dedicated control channel easily congested by regular updates of periodic safety messages and broadcast storm from event-driven safety messages. The congestion control approach is one of the best solutions to solve congestion wireless communication channel in VANETs. The proposed framework of congestion control will scan the messages queues and monitor channel communications based on defined threshold. A priority-based EDF scheduling algorithm was also proposed for congestion control. The purpose of algorithm is to schedule packets with same high priority in order to provide reliability performance for event-driven safety messages [25] also proposed to develop a new rebroadcasting scheme to reduce the channel load.

Table 3. IoT based VANET systems

\begin{tabular}{|c|c|}
\hline System & Main idea \\
\hline $\begin{array}{l}\text { Software Defined Networking-based } \\
\text { Vehicular Adhoc Network with Fog }\end{array}$ & Emerged SDN with Fog Computing in VANET. \\
\hline Computing & \\
\hline $\begin{array}{llr}\text { VANET based } & \text { Integrated } \\
\text { Framework for Smart } & \text { Accident } \\
\text { Management System } & \end{array}$ & Integrated IoT and $\mathrm{A} *$ algorithm to VANET. \\
\hline $\begin{array}{l}\text { Game theoretic approach on Real- } \\
\text { time decision making for IoT based } \\
\text { traffic light control }\end{array}$ & $\begin{array}{l}\text { Using game theoretic approach with IoT in } \\
\text { VANET. }\end{array}$ \\
\hline $\begin{array}{l}\text { A Congestion Control Sy stem Based } \\
\text { on VANET for Small Length Road }\end{array}$ & Using the structure of BUS-VANET. \\
\hline Converging VANET with Vehicular & Discuss the possible components of VANET and \\
\hline $\begin{array}{l}\text { Cloud Networks to reduce the } \\
\text { Traffic Congestions: A Review }\end{array}$ & $\begin{array}{l}\text { IoT (Vehicular Cloud Computing) to solve } \\
\text { traffic flow. }\end{array}$ \\
\hline $\begin{array}{l}\text { Congestion Control Framework for } \\
\text { Disseminating Safety Messages in } \\
\text { Vehicular Ad-Hoc Networks } \\
\text { (VANETs) }\end{array}$ & $\begin{array}{l}\text { Proposed a scalable congestion control based in } \\
\text { defined threshold and EDF scheduling algorithm. }\end{array}$ \\
\hline
\end{tabular}

Indonesian J Elec Eng \& Comp Sci, Vol. 12, No. 1, October $2018: 218$ - 224 


\section{CONCLUS ION}

This paper presents the review on IoT-based VANETs to overcome the drawbacks of the traditional initial VANET. IoT as the alternative, is expected to support the future ITS with the ultimate goal of making driving safer and more enjoyable. However, there are needs of further research on this topic since it is still insufficient.

Upcoming ITS based IoT can offer real-time required responses to vehicles drivers [5]. Integrating IoT in VANET is expected to ensure more flexibility, low-latency and high-bandwidth communication. Expectation of creating the future vision enhanced vehicular networks to enhance the traffic management. Merging these technologies in ITS will be the vital basis for the revolution towards the global automation environment $[5,18]$.

\section{ACKNOWLEDGMENT}

The authors would like to thank the Faculty of Computer and Mathematical Sciences (FSKM), Universiti Teknologi Mara (UiTM) for sponsoring this research.

\section{REFERENCES}

[1] N. B. Truong, G. M. Lee, and Y. G. Doudane, "Software Defined Networking-based Vehicular Adhoc Network with Fog Computing," 2015, IEEE.

[2] E. C. Eze, S. Zhang and J. C. Eze, "Advances in Vehicular Ad-Hoc Networks (VANETs): Challenges and Road map for Future Development," 2016.

[3] W. Liang, Z. Li, H. Zhang, S. Wang, and R. Bie, "Vehicular Ad Hoc Networks: Architectures, Research Issues, Methodologies, Challenges, and Trends," 2015, International Journal of Distributed Sensor Networks, Volume 5.

[4] A. Dua. N. Kumar, and S. Bawa, "QoS-Aware Data Dissemination for Dense Urban Regions in Vehicular Ad Hoc Networks," 2015, Mobile Network Application (2015) 20:773-780.

[5] M.S. Talib, B. Hussin, and A. Hassan, "Converging VANET with Vehicular Cloud Networks to reduce the Traffic Congestions: A review," 2017, International Journal of Applied Engineering Research, Volume 12, Number 21, pp. 10646-10654.

[6] A. Nandan, S. Das, G. Pau, and M. Gerla, "Cooperative downloading in vehicular ad hoc wireless networks," IEEE Conf. WONS, Switzerland, Jan. 2005, pp. 32-41.

[7] O. Riva, T. Nadeem, C. Borcea, and L. Iftode, "Context-aware migratory services in ad hoc networks" IEEE Trans. Mobile Comput., vol. 6, no. 12, pp. 1313-1328, December, 2007.

[8] BBCR Group, Basic VANET System, 2006, Electrical and Computer Engineering, University of Waterloo.

[9] C. Chatrapathi, M. N. Rajkumar, and V. Venkatesakumar, "VANET based Integrated Framework for Smart Accident Management System," 2015, International Conference on Soft-Computing and Network Security.

[10] J. Morgan, “A Simple Explanation Of The Internet Of Things," 2014.

[11] J. Zhou, Z. Cao, X. Dong, and A. V. Vasilakos, "Security and Privacy for Cloud-Based IoT: Challenges, Countermeasures, and Future Directions," 2017, IEEE.

[12] W. He, G. Yan., and L. D. Xu, "Developing Vehicular Data Cloud Services in the IoT Environment," IEEE TransactionsOn Industrial Informatics, Vol. 10, No.2, May 2014.

[13] N. B. K. Hoai and D. Camacho, "Game theoretic approach on Real-time decision making for IoT-based traffic light control," WILEY, 2016.

[14] R. Jain, "A Congestion Control System Based on VANET for Small Length Roads," Annals of Emerging Technologies in Computing (AETiC), Vol. 2, No. 1, 2018.

[15] Z. Bouyahia, H. Haddad, N. Jabeur, and S. Derrode, "Real-Time Traffic Data Smoothing from GPS Sparse Measures Using Fuzzy Switching Linear Models," in Procedia Computer Science, 2017, vol. 110, pp. 143- 150

[16] A. Perova, "Methods of Placement of Business Tourism Centers in Large Cities as Means Providing Traffic Safety (on the Example of St. Petersburg)," in Transportation Research Procedia, 2017, vol. 20, no. September 2016, pp. 487-492.

[17] J. Contreras, S. Zeadally, and J. A. Guerrero-Ibanez, "Internet of Vehicles: Architecture, Protocols, and Security," IEEE Internet Things J., vol. 4662, no. c, pp. 1-1, 2017.

[18] E. Burcoci, "From Vehicular Adhoc Networks to Internet of Vehicles," NexComm 2017 Conference.

[19] S. A. Ben Mussa, M. Manaf, K. Z. Ghafoor, and Z. Doukha, "Simulation tools for vehicular ad hoc networks: A comparison study and future perspectives," 2015 International Conference on Wireless Networks and Mobile Communications (WINCOM), Oct. 2015.

[20] J. Cheng. J. Cheng. M. Zhou. F. Liu. S. Gao and C. Liu (2015). "Routing in internet of vehicles: A Review," IEEE Transactions on Intelligent Transportation Systems 2017, 16(5), pp. 2339-2352.

[21] W. Chao, Mohammed A. Quddus and S. G. Ison. "The effect of traffic and road characteristics on road safety: A review and future research direction”. Safety Science, 57, 264-275, 2017.

[22] R. Bauza, J. Gozalvez and J. Sanchez-Soriano. "Road traffic congestion detection through cooperative Vehicle-toVehicle communications," Proceedings - Conference on Local Computer Networks, LCN, 606-612, 2010.

[23] Y. D. Mohamad, M. S. Z. Abidin, S. J. Elias and Z. Zainol. "Optimizing Congestion Control for Non Safety Messages in VANETs Using Taguchi Method,”2017. 
[24] S. A. B. Mussa, M. Manaf, K. Z. Ghafoor, Z. Doukha. "Simulation Tools for Vehicular Ad Hoc Networks: A Comparison Study and Future Perspectives", IEEE 2015.

[25] M. Y. Darus and K. A. Bakar. "Congestion Control Framework for Disseminating Safety Messages in Vehicular Ad-Hoc Networks (VANETs)," International Journal of Digital Content Technology and its Applications. Volume 5, Number 2, February 2011.

[26] M. D. Felice, I. V. Calcagni, F. Pesci, F. Cuomo and A. Baiocchi. "Self-Healing Infotainment and Safety Application for VANET dissemination", IEEE ICC 2015.

[27] A. Wahid, H. Yoo, and D. Kim, "Unicast geographic routing protocols for inter-vehicle communications: a survey," in Proceedings of the 5th ACM Workshop on Perfor Mance Monitoring and Measurement of Heterogeneous Wireless and Wired Networks (PM2HW2N '10), pp. 17-24, New York, NY, USA, 2010.

[28] H. A. Omar ; N. Lu ; W. Zhuang. "Wireless access technologies for vehicular network safety applications," IEEE Network, Volume: 30 Issue: 4, 2016.

[29] L.Wischhof and H. Rohling, "Congestion Control in Vehicular Ad Hoc Networks.", In Proceeding of International Conference on Vehicular Electronics and Safety, pp. 58-63, 2005.

[30] R. Khatoun and S. Zeadally. Cybersecurity and privacy solutions in smart cities. IEEE Communications Magazine, 55(3), 51-59, 2017.

[31] H. Menouar, I. Guvenc, K. Akkaya, A. S. Uluagac, A. Kadri and A. Tuncer. "UAV-Enabled Intelligent Transportation Systems for the Smart City: Applications and Challenges," IEEE Communications Magazine Volume: 55, Issue: 3, 2017.

[32] S. Kohli, B. Kaur, S. Bindra. "A comparative study of Routing Protocols in VANET," The International Sy mposium on Computer Science 2010. 